Method A total of 50 mothers who have hospitalized infants in NICU, included in this study and the social support they perceived, and the their anxiety and depression levels were analyzed using "Multidimensional Scale of Perceived Social Support Scale" and Hospital Anxiety and Depression Scale (HAD scale), respectively.

Results Perceptions of total social support and the social support provided by friends in the mothers increased as the infants' birth weight decreased; the levels of total perceived social support and the perceived social support from families and spouses in the mothers getting pregnant with assisted conception techniques (ACT) were greater, compared with the mothers of spontaneous conception. The scores of total perceived social support and the perceived social support from families and spouses in depressed mothers were found to be lower than those in otherwise healthy mothers.

Conclusion It was concluded in our study that the mothers of the hospitalized infants in the neonatal intensive care unit required social support provided especially by the spouses and the families, disclosing the relationship of inadequate perception of such supports with development of depression.

\section{DO WE REALLY NEED TO REQUEST THAT BLOOD INVESTIGATION?}

doi:10.1136/archdischild-2012-302724.1765

AM Kulkarni, R Kinnapiran, N Kennea. Neonatology, St George's Hospital NHS Trust, London, UK

Blood investigations form an important aspect of patient management. Rationalisation of every test requested is needed to ensure optimum use of available resources. Wide variation in estimates of inappropriate laboratory use (4.5-95\%) has been reported in literature. Hospitalisation beyond 7 days, complex cases, level of staff training, lack of awareness of costs are factors contributing to laboratory over utilisation.

We assessed our blood investigation ordering practices in order to evaluate unnecessary investigations and clinical variance. We aimed to develop a standard framework for ordering investigations in preterm babies.

This retrospective study (Jan-June 2010) evaluated blood investigations requested in first 14 days of life on the all the babies born $<30$ weeks gestation and admitted to our neonatal unit. These data amounted to $\sim 5 \%$ of bed days on the unit over the study period. We created a standard investigation model for preterm babies and compared the findings with the standard.

37 babies were included in the study with total 479 cot days which represent $4.3 \%$ of unit cot days per year. We observed that we were requesting nearly $40 \%$ more tests as compared to standard. We estimated potential savings of $£ 1000 \mathrm{GBP}$ for only $4.3 \%$ of total cot days every year in our unit.

Following audit was presented in our departmental meeting for the education of junior members of the staff and to raise the awareness of the costs of investigations and the need to reduce unnecessary testing. We recommended colour coding of pathology forms according to cost.

\section{RANDOMISED CONTROLLED TRIAL OF POLYETHYLENE BAG AND EXOTHERMIC MATTRESS VERSUS POLYETHYLENE BAG ALONE FOR THERMOREGULATION IN PRETERM INFANTS AT BIRTH}

doi:10.1136/archdischild-2012-302724.1766

1,2,3LK McCarthy, ${ }^{1,2}$ EJ Molloy, ${ }^{1}$ AR Twomey, ${ }^{1} \mathrm{JF}$ Murphy, ${ }^{1,2,3} \mathrm{CPF}$ 0'Donnell. 'Department of Neonatology, The National Maternity Hospital; ${ }^{2}$ The National Children's Research Centre, Crumlin; ${ }^{3}$ University College Dublin, Dublin, Ireland

Aims To determine whether placing very preterm infants in polyethylene bags( $\mathrm{PB})$ and on exothermic mattresses(EM) in the delivery room(DR) results in more infants with temperatures of $36.5-37.5^{\circ} \mathrm{C}$ on NICU admission.

Methods Infants $<31$ weeks' were randomised to treatment with or without EM in DR. All infants were placed in $\mathrm{PB}$ under radiant heaters and were transferred to the NICU in transport incubators. Infants' rectal temperature was measured on admission. We estimated we would need to recruit 118 infants.

Results The external data safety monitoring committee recommended stopping recruitment after analyzing data from 59 infants due to a significant difference in primary outcome between the groups. We present data for 72 infants enrolled when this recommendation was made. Fewer infants randomised to EM had temperatures within the target range $[41 \% \vee 77 \%, p=0.002]$ and more had admission temperature $>37.5 \mathrm{C}$ [ $46 \%$ v $17 \%, \mathrm{p}=0.009]$.

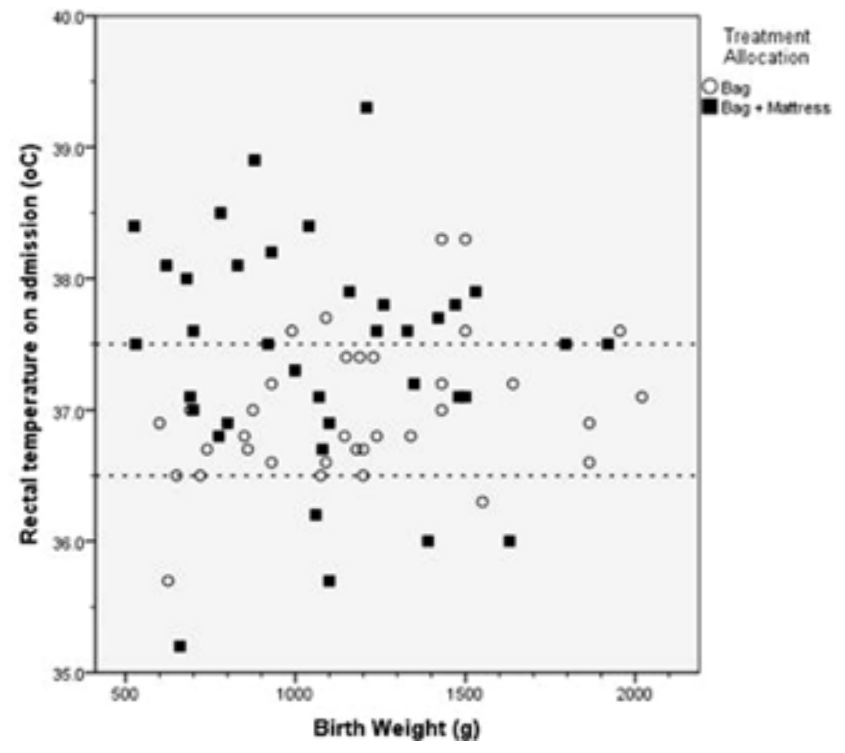

Abstract 1766 Figure 1

Abstract 1766 Table 1 Results

\begin{tabular}{lccc}
\hline [Data are * mean $(\mathbf{S D})$ or \#n (\%)] & BAG \& MATTRESS (N=37) & BAG (N=35) & P value \\
\hline Gestational age $(w k s)^{*}$ & $28(2)$ & $28(2)$ & 0.584 \\
Birth weight $(\mathrm{g})^{*}$ & $1085(360)$ & $1194(386)$ & 0.222 \\
Adm rectal temp 36.5-37.5(C)\# & $15(41)$ & $27(77)$ & 0.002 \\
Adm rectal temp (C) & $37.4(0.9)$ & $37.0(0.5)$ & 0.017 \\
Adm rectal temp>37.5C\# & $17(46)$ & $6(17)$ & 0.009 \\
Adm rectal temp<36.5C\# & $5(14)$ & $2(6)$ & 0.264 \\
\hline
\end{tabular}

Conclusions In newborn very preterm infants, using EM in addition to $\mathrm{PB}$ in the $\mathrm{DR}$ resulted in more infants with temperatures outside normal range and more hyperthermia on admission to NICU.

\section{VALIDATION OF THE DUTCH 60 MONTHS AGES AND STAGES OUESTIONNAIRE (ASO)}

doi:10.1136/archdischild-2012-302724.1767

1J Hornman, ${ }^{1} \mathrm{JM}$ Kerstjens, ${ }^{2} \mathrm{AF}$ de Winter, ${ }^{1} \mathrm{AF}$ Bos, ${ }^{2} \mathrm{SA}$ Reijneveld, Lollipop Study. ${ }^{1}$ Department of Neonatology, Beatrix's Children Hospital, University Medical Centre Groningen; ${ }^{2}$ Department of Health Sciences, University Medical Centre Groningen, University of Groningen, Groningen, The Netherlands

Introduction The Ages and Stages Questionnaire (ASQ) is currently the most widely used parent-completed developmental screener. Psychometric properties of the Dutch ASQ 60-months version (Dutch_ASQ-60) have not yet been assessed. 